\title{
QCM-D for Non-Destructive Real-Time Assessment of Pseudomonas aeruginosa Biofilm Attachment to the Substratum during Biofilm Growth
}

\author{
Revised and resubmitted to:
}

Colloids and Surfaces B: Biointerfaces

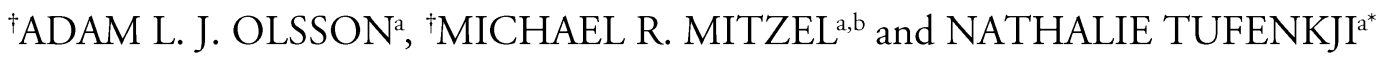

${ }^{a}$ Department of Chemical Engineering,

${ }^{b}$ Department of Natural Resource Sciences,

McGill University, Montreal, Quebec, H3A OC5, Canada

adam.olsson@mail.mcgill.ca,mike.mitzel@mail.mcgill.ca,nathalie.tufenkji@mcgill.ca

"Corresponding Author. Phone: (514) 398-2999; Fax: (514) 398-6678; E-mail: nathalie.tufenkji@mcgill.ca

${ }^{\dagger}$ These authors contributed equally to this work. 
- 2 - 


\begin{abstract}
Quartz crystal microbalance with dissipation monitoring (QCM-D) was used to investigate initial adhesion and subsequent biofilm growth of wild-type Pseudomonas aeruginosa PAO1 and a pilideficient $(\Delta$ pilA) mutant PAO1 strain. Clean, sterilized, silica-coated QCM-D crystals were pre-coated with lysogeny broth (LB), seeded with a PAO1 strain and allowed to grow for $20 \mathrm{~h}$ at $37^{\circ} \mathrm{C}$ in fresh LB injected at $100 \mu \mathrm{L} / \mathrm{min}$. QCM-D signals obtained for the wild-type PAO1 strain during the seeding period depict a large positive frequency shift that returns to baseline after -20 min that is absent in the $\Delta$ pilA mutants, suggesting a dynamic pili-mediated attachment event for the wild-type PAO1 strain. During the subsequent growth period, significant and characteristic differences in the acquired QCM-D signals were observed between the wild-type and the $\Delta$ pilA mutant. Confocal laser scanning microscopy (CLSM) of the biofilm on the crystal surface showed that these differences could not be explained by differences in the extent of biofilm growth alone. When interpreted according to a coupled resonance model, the QCM-D observations suggest that pili are essential for coupling the developing biomass to the sensor surface. Total internal reflection fluorescence microscopy (TIRF) supports the hypothesis that the characteristic QCM-D signal is indicative of a dynamic attachment event, mediated by pili cell surface appendages pulling the wild-type PAO1 closer to the surface during the seeding period. We show that QCM-D offers direct, non-disruptive, in situ measurements of biofilm-substrate attachment. This technique has the potential to improve the current understanding of biofilm formation phenomena.
\end{abstract}

Keywords

QCM-D, biofilm, pili, TIRF, Pseudomonas aeruginosa, interface 


\section{Introduction}

Bacterial attachment and subsequent biofilm growth occurs on virtually all wet surfaces. Biofilms are generally undesirable when formed on man-made surfaces, as they may cause, for instance, intractable biomaterial related infections [1], food contamination [2, 3] and pipeline plugging or corrosion [4]. However, biofilms are also essential in settings such as wastewater treatment, bioremediation, or as a symbiotic component of an organism's internal microbiome [5-7]. Thus, extensive research efforts have been directed towards understanding the bacterial attachment and biofilm formation processes to identify strategies for controlling biofilm development.

Approaches such as confocal laser scanning microscopy (CLSM), biofilm disruption combined with staining assays and/or CFU counts, low load compression testing and molecular expression assays have provided valuable information on how biofilm morphology [8], biomass [9], rheological properties [10] and gene or protein expression [11, 12] change during biofilm development; however, none of these methods provide insight into surface attachment mechanisms. Atomic force microscopy [13, 14], optical tweezers [15] and flow-displacement devices [16] are methods used to study the force required to detach bacterial cells from a surface [17]. These techniques have improved our knowledge about the dynamic interfacial phenomena at the level of individual bacteria, but this information is not easily extrapolated to the level of a microbial community within a biofilm structure.

Microorganisms within a biofilm communicate among themselves to develop into organized, three-dimensional structures. Biofilm formation by $P$. aeruginosa PAO1 has been extensively studied and is decidedly a complex process involving a variety of genetic cues, metabolic changes and morphological stages [12, 18-21]. How these events affect the attachment properties of biofilms is poorly understood and available information is generally limited to forces 
required to remove biofilm as measured by destructive micromanipulation techniques (e.g., scraping) [17]. Establishing alternative methods to study how biofilms are attached to surfaces is thus desirable as such information can be expected to aid in the development of antifouling surfaces and biofilm removal strategies.

One technique that has the potential to non-disruptively and non-destructively provide information about the biofilm-substrate contact interface is the quartz crystal microbalance (QCM). The QCM senses mass attachment to an oscillating quartz sensor in terms of shifts in its resonance frequency. By relating a negative frequency shift to an area-averaged mass via the Sauerbrey relation [22], it is generally used as a 'microbalance' (hence its name) with sensitivity of $\mathrm{ng} / \mathrm{cm}^{2}$. For this reason, the QCM has been suggested as a method for real-time assessment of biomass during biofilm growth [23, 24]. Indeed, many studies on biofilm growth in QCM report that the sensor resonance frequency shifts in the negative direction [25-31], in accordance with the conventional Sauerbrey relation. However, some studies have reported that biofilm growth shifted the resonance frequency in the positive direction [32-35], which is in disagreement with the Sauerbrey relation.

Positive frequency shifts have been explained to occur when a large mass (e.g., micronsized particles, including bacteria) attaches via "point contacts" and these point contacts are too weak for the attached mass to fully couple to the sensor surface and thereby be laterally displaced by the crystal oscillation $[36,37]$. Instead, the mass, now withheld in space by its own inertia and thus not being laterally displaced, exerts a restoring force which adds to the stiffness of the crystal thereby shifting the resonance frequency in the positive direction [38]. The relative difference in lateral displacement between the sensor surface and the attached mass can be modelled as a "coupled resonance" [39] which briefly means that the resulting shift in sensor resonance 
frequency shift is governed by the inherent resonance frequency of the attached sample which, in turn, depends on the mechanical properties of its contact with the surface. The coupled resonance model has been applied to the initial adhesion of bacteria to investigate, for instance, the effect of ionic strength on contact stiffness of Streptococcus salivarius strains carrying fibrils of different lengths [40] or the effect of cell surface hydrophobicity on contact stiffness of Pseudomonas aeruginosa attachment to hydrophobic and hydrophilic surfaces [41].

Since the coupled resonance model applies to adhering bacteria and because biofilms are essentially linked to the surface via these initial surface colonizers, we hypothesized that QCM offers a unique non-destructive approach to probe the surface attachment of biofilms. We employed a QCM with dissipation monitoring (QCM-D) to examine bacterial adhesion and subsequent biofilm growth and development of wild-type $P$. aeruginosa $\mathrm{PAO} 1$ and a pili-deficient $\Delta$ pilA mutant PAO1 strain, with the specific aim to determine the role of pili on the surface attachment of biofilms. For further data corroboration, CLSM was used to evaluate biofilm biomass and morphology and total internal reflection fluorescence microscopy (TIRF) was used to investigate the impact of pili presence on initial bacterium surface approach.

\section{Materials and Methods}

\section{Bacterial Species and Inoculum Preparation}

Wild-type Pseudomonas aeruginosa PAO1 (ATCC \#BAA-47) and a pili knockout mutant ( $\Delta$ pilA) were streaked from a $-80{ }^{\circ} \mathrm{C}$ stock onto a lysogeny broth (LB; Lauria formulation) agar plate and incubated overnight at $35 \pm 2^{\circ} \mathrm{C}$. Without refrigeration, $200 \mathrm{~mL}$ of LB was inoculated and incubated at $35 \pm 2^{\circ} \mathrm{C}$ and $130 \mathrm{rpm}$ in a $500 \mathrm{~mL}$ culture flask. For the overnight biofilm growth, the flask was prepared with sterile tubing and connections. Inoculum density was monitored by viable cell 
counts and ranged between $1 \times 10^{9}$ to $6 \times 10^{9} \mathrm{CFUs}$ for the wild-type and $2 \times 10^{9}$ to $1 \times 10^{10}$ for the $\Delta$ pilA mutant PAO1 strains.

\section{QCM-D Cleaning, Seeding and Growth}

QCM-D silica crystals were cleaned with $2 \%$ SDS by sonication for 2 min, overnight soak, followed by an additional 2 min of sonication. The same procedure was then done using $2 \%$ Hellmanex ${ }^{\mathrm{TM}}$. Crystals were then dried and UV irradiated for $20 \mathrm{~min}$ before mounting into the QCM-D chamber. QCM-D temperature control was set to $37^{\circ} \mathrm{C}$. The QCM-D setup (i.e., tubes, junctions, crystals and chambers) was flushed with $70 \%$ ethanol for 20 min prior to experimentation. Sterile LB was then fed through the QCM-D until a stable baseline was reached for at least $10 \mathrm{~min}$. Bacterial inoculum was then injected for $1 \mathrm{~h}$, followed by fresh LB for $20 \mathrm{~h}$. The flow rate was set to $100 \mu \mathrm{L} / \mathrm{min}$. Control experiments conducted with sterile $\mathrm{LB}$ alone confirmed the lack of contamination in the QCM-D system (i.e., no biofilm growth detected).

\section{QCM-D Data Analysis}

The acquired QCM-D data during biofilm growth was interpreted within the context of the coupled resonance model [37-39]. The model ascribes the measured frequency shift to the lateral displacement of the attached sample relative to that of the oscillating sensor surface. The sample's lateral displacement is further ascribed to its inherent resonance frequency $f$ which is governed by its mass $m$ and coupling constant $k$ (i.e., contact stiffness) according to:

$$
f=\frac{1}{2 \pi} \sqrt{\frac{k}{m}}
$$


The frequency shift of this coupled resonance is negative if the coupling constant is large enough to provide a sample resonance frequency above the crystal resonance frequency; otherwise it is positive. Accordingly, a change in the sample's mass $m$ or coupling constant $k$ may, as long as the sample's resonance frequency falls within the observable resonance frequencies of the QCM (normally within the $\mathrm{MHz}$ range), cause a transition from a negative to positive frequency shift and/or vice versa [36]. This transition is accompanied with a peak in dissipative energy losses, measured either in terms of the crystal oscillation decay rate (dissipation, $D$ ) or the width of the oscillation peak (bandwidth, $\Gamma$ ). In this study, we limited our analysis to the frequency and dissipation shifts acquired at the sensor's third overtone resonance frequency of $15 \mathrm{MHz}$.

\section{Confocal Imaging and Biomass Estimates}

Crystals were removed from the QCM-D after seeding $(t=1 \mathrm{~h})$, before $(t=4 \mathrm{~h})$ and after $(t=6 \mathrm{~h})$ a characteristic fluctuation in the measured signals (see Results section), as well as at the end of the experiment $(t=20 \mathrm{~h})$. Biofilm-coated crystals were stained using FilmTracer ${ }^{\mathrm{TM}} \mathrm{FM}{ }^{\circledR}$ 1-43 Green Biofilm Cell Stain (Molecular Probes $\left.{ }^{\circledR}\right)$ following the manufacturer's instructions. A Zeiss LSM 510 META confocal laser scanning microscope (CLSM) equipped with an argon laser (488 $\mathrm{nm}$ ) was used to image the crystal surface following staining using a bandpass filter of 530-600 nm. Images were acquired using Zen $2000^{\mathrm{TM}}$ software. A minimum of 6 stacks ( collected from each QCM-D crystal at locations near the middle of the crystal. Biomass estimates were calculated using COMSTAT2 software using Otsu's method for automatic thresholding for all images.

Total Internal Reflection Fluorescence Microscopy (TIRF) 
For TIRF experiments, $5 \mathrm{~mL}$ of the overnight broth culture was combined with $250 \mu \mathrm{L}$ of FM® 1-43 stain (Molecular Probes), vortexed and left to stand for $15 \mathrm{~min}$. Cell-stain mixture was centrifuged at $3000 \times g$ for 5 min and resuspended in $10 \mathrm{~mL}$ of fresh $\mathrm{LB}$. $500 \mu \mathrm{L}$ of fresh LB was added to a glass bottom Petri dish and $20 \mu \mathrm{L}$ of stained cells added. A TIRF-Discovery Platform (Spectral Applied Research, Exton, PA) with a 63X Plan Apochromat, NA = 1.47 Oil, DIC from Leica, a 488 Diode laser with FLCRtm control and a Hamamatsu ImagEMX2 EMCCD Digital

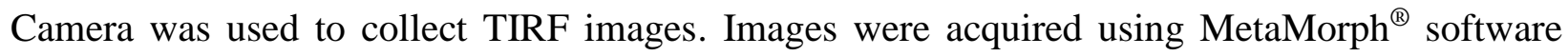
(Molecular Devices Corporation, Downingtown, PA) with an exposure time of $0.15 \mathrm{~s}$, a penetration depth of $200 \mathrm{~nm}$ and image acquisition every 1 s. Image intensities were further analyzed using ImageJ v1.48. A minimum of 50 individual bacteria per strain, divided over two separately grown cultures, were selected by defining a rectangular area just large enough to encompass the bacterium of interest. The average intensities of these rectangular areas were measured for each image within the collected time series.

As TIRF fluorescence intensity decreases exponentially with distance from the glass surface, the change in bacterium-surface separation distance is linearly proportional to the natural logarithm of the fluorescence intensity change $\left(\ln \left(I / I_{0}\right)\right)[42]$. For a more quantitative assessment of the bacterium-surface approach we used a least-squares regression analysis to fit an exponential function to the florescence intensity data according to [43]:

$$
\ln \left(I / I_{0}\right)_{t}=\ln \left(I / I_{0}\right)_{\infty}\left(1-e^{-(t / \tau)}\right)
$$

where $I_{0}$ is the bacterium fluorescence intensity as it first appears in the image sequence (i.e., at $t$ $=0), I$ indicates the bacterium fluorescence intensity at its surface residence time $t$, and $\tau$ the characteristic bacterium surface approach time constant. 


\section{Results}

\section{QCM-D Monitoring of Biofilm Growth}

Figure 1 shows representative frequency shifts $(\Delta f)$ (a) and dissipation shifts $(\Delta D)$ (b) of the $3^{\text {rd }}$ overtone during an initial seeding period (I) and the subsequent biofilm growth period (II) for wildtype $P$. aeruginosa $\mathrm{PAO} 1$ and the $\mathrm{PAO} 1 \Delta$ pilA mutant. Figure 2 presents the magnitude of $\Delta f$ and $\Delta D$ at four time points $(1 \mathrm{~h}, 4 \mathrm{~h}, 6 \mathrm{~h}$ and $20 \mathrm{~h})$ selected for biofilm CLSM imaging for all measurements. Wild-type $P$. aeruginosa PAO1 initially displayed a rapid increase in $\Delta f$ that reached a positive frequency shift of $\sim 100 \mathrm{~Hz}$ approximately $10 \mathrm{~min}$ into seeding and was followed by a sudden decrease in $\Delta f$. This decrease in $\Delta f$ eventually brought the frequency down below the initial baseline value of $0 \mathrm{~Hz}$ (i.e., to a negative frequency shift). The sudden drop to a negative $\Delta f$ was accompanied with a peak value of $\sim 50 \times 10^{-6}$ in $\Delta D$, whereby $\Delta D$ remained positive after the peak. Positive $\Delta f$ for initial $P$. aeruginosa PAO1 deposition has been reported previously [41]. After the overnight culture was replaced by fresh growth medium for the growth phase of the experiment, the negative $\Delta f$ slowly started to increase and soon turned positive again. The frequency shift continued its gradual increase until the termination of the experiment at $20 \mathrm{~h}$. Similarly, $\Delta D$ demonstrated a continuous and gradual increase until the completion of the experiment. A notable and defining feature of $P$. aeruginosa PAO1 biofilm growth on the QCMD crystal was a fluctuation in both $\Delta f$ and $\Delta D$ that occurred consistently at a time between 4-6 hours of incubation. This behavior was present in all experiments, although more or less pronounced in some cases and occurring at slightly different incubation times.

The $\Delta f$ and $\Delta D$ response of $P$. aeruginosa PAO1 $\Delta$ pilA mutant deposition and biofilm growth was dramatically different to that of the wild-type $P$. aeruginosa PAO1. Instead of a 
significant peak in positive $\Delta f$ and $\Delta D$ values during initial seeding, the $\Delta$ pilA mutant yielded only a small positive $\Delta f$ that reached and maintained a value of only a few $\mathrm{Hz}$ and the $\Delta D$ response was indistinguishable from the baseline value. Similar to the wild-type $P$. aeruginosa PAO1, upon replacing the overnight culture with fresh growth medium, both the positive $\Delta f$ and $\Delta D$ slowly increased in magnitude for the remainder of the experiment, albeit at a much slower rate and degree than the wild-type P. aeruginosa PAO1.

\section{Microscopic Observation of Bacteria and Biofilm}

Figure 3 shows representative maximum intensity CLSM images of $P$. aeruginosa PAO1 (a) and the PAO1 $\Delta$ pilA mutant (b) biofilm development throughout the $20 \mathrm{~h}$ growth period. Directly after the seeding step $(t=1 \mathrm{~h})$, the bacteria had adhered as single cells and were evenly distributed over the surface for both strains. After $3 \mathrm{~h}$ of growth in fresh nutrient broth (i.e., at $t=4 \mathrm{~h}$, before the fluctuation in the QCM-D signal observed for the wild-type $P$. aeruginosa PAO1 in Figure 1) both strains had started to multiply and form microcolonies, but striking morphological differences emerged between the two, consistent with those reported by Klausen et al. [21]. While $P$. aeruginosa PAO1 showed signs of migration to cover the bare surface area in between the formed microcolonies, the pili-deficient $\triangle$ pilA mutant PAO1 instead appeared to continue to grow in the form of colonies, i.e. multiplying but not moving to cover the surface. After five hours of growth (i.e., at $t=6 \mathrm{~h}$, following the fluctuation in the QCM-D signal observed for $P$. aeruginosa PAO1 in Figure 1), wild-type P. aeruginosa PAO1 appeared to have continued its migration to cover any remaining available surface area. Conversely, the $\Delta$ pilA mutant (that did not display fluctuations) appeared to have continued to grow in localized patches, without much movement to cover empty spaces. Finally, after 20 h, P. aeruginosa PAO1 had formed a homogeneous sheet-like biofilm 
layer, in stark contrast to the pili-deficient $\triangle$ pilA PAO1 mutant that had formed a mushroom-like biofilm with localized patches of cells.

\section{Biofilm Biomass Quantification}

The biomass determined by COMSTAT analysis of the CLSM images used for qualitative assessment of biofilm morphology is presented in Figure 4 for P. aeruginosa PAO1 (a) and $\triangle$ pilA mutant (b). Both strains demonstrated a similar gradual increase in biofilm mass during the first 6 hours of growth. After 20 hours, wild-type $P$. aeruginosa PAO1 showed a larger average biomass than the $\Delta$ pilA mutant, although a large variance precludes distinguishing the two biofilms based on biomass alone. Differences in biomass of the two biofilm types can thus not account for the distinct differences in measured $\Delta f$ and $\Delta D$. The large variance in biomass for wild-type $P$. aeruginosa $\mathrm{PAO} 1$ is to some extent an experimental artifact associated to challenging microscopy sample preparation for this specific experimental condition (see Figure S1 Supporting Information for a representative image showing the $P$. aeruginosa PAO1 biofilm folding onto itself during removal of the QCM crystal from the measurement chamber).

Average values of $\Delta f$ and $\Delta D$ plotted against average biomass estimates at four time points $(1 \mathrm{~h}, 4 \mathrm{~h}, 6 \mathrm{~h}$ and $20 \mathrm{~h}$ ) for the wild-type P. aeruginosa PAO1 and $\Delta$ pilA mutant biofilms are presented in Figure 5. The comparison of $\Delta f$ and $\Delta D$ with biomass for the wild-type $P$. aeruginosa PAO1 displays a trend of increasing signal with increasing biomass. By comparison, the $\Delta$ pilA mutant shows little to no change in $\Delta f$ or $\Delta D$ with increasing biomass, indicating that this mass is not well coupled to the QCM-D crystal surface.

TIRF Imaging and Analysis 
Representative examples of TIRF fluorescence intensities for individual wild-type and pilideficient PAO1 cells as a function of bacterium-surface residence time are shown in Figure 6a together with the outcome of the least-squares regression analysis according to equation 2 . Note that the initial fluorescence intensity values $\left(I_{0}\right)($ i.e., at $t=0)$ were obtained from the time point where an individual bacterium first appeared (i.e., the first image within the sequence), and the intensity change is thus normalized to the furthest measured bacterium-surface separation distance (a representative series of TIRF images of an approaching bacterium is included in Figure S2 Supporting Information).

The outcome of the least-squares regression analysis of 50 individual bacteria for each strain is presented in the format of histograms in Figure 6b-e. For both strains, the final change in fluorescence intensity (i.e., $\left.\ln \left(I / I_{0}\right)_{\infty}\right)$ distribution was centered around a value of 1 . While the intensity distribution for the $\Delta$ pilA mutant appears slightly skewed towards values below 1 (Figure 6d), the intensity distribution for the PAO1 wild-type strain appears to be skewed towards values larger than 1 (Figure 6b), suggesting a shorter average bacterium-surface separation distance and/or larger average bacterium-surface contact area for PAO1 wild-type strain than for the $\Delta$ pilA mutant strain. The characteristic bacterium-surface approach time constant $\tau$ showed two distinctly different distributions for the two strains. While the wild-type P. aeruginosa PAO1 was found to be distributed around approach time constants of $1.5 \mathrm{~s}$ (Figure 6c), more than $50 \%$ of the pilideficient $\Delta$ pilA mutant cells reached their final bacterium-surface distance within the first $0.5 \mathrm{~s}$ (Figure 6e).

\section{Discussion}

We have studied $P$. aeruginosa PAO1 attachment to silica and subsequent biofilm growth in a QCM-D with the aim of distinguishing between the wild-type strain P. aeruginosa PAO1 and a 
pili-deficient $P$. aeruginosa $\mathrm{PAO} \triangle \Delta$ pilA mutant strain based on their respective biofilm surface attachment properties. While QCM is generally considered a microbalance able to quantify biofilm biomass by means of negative frequency shifts of the quartz crystal sensor, both $P$. aeruginosa strains studied herein shifted the sensor's resonance frequency in the positive direction during biofilm growth. The biofilm-induced positive frequency shifts presented herein thus demonstrate that biofilm growth in QCM does not follow conventional mass loading theory (i.e. Sauerbreytype loading). Rather, these results suggest that biofilm growth in QCM-D follows a coupled resonance model.

The coupled resonance model, previously applied to interpret initial bacterial attachment in QCM-D [36, 37, 41] predicts positive frequency shifts for weakly attached masses that are larger than the ambient liquid shear wave penetration depth (induced by the crystal oscillation). Positive frequency shifts have also been explained in terms of "film resonance" which is due to the deformation of homogeneous, thick (few hundred nanometers) and viscoelastic molecular films $[44,45]$. Bacteria are "particle-like" and the $P$. aeruginosa PAO1 and the $P$. aeruginosa $\Delta$ pilA strains are not expected to be significantly different in terms of either their viscoelastic properties or size; but they are expected to be significantly different in their surface attachment properties because of the absence or presence of pili surface appendages. We therefore propose that the coupled resonance model, earlier applied when initial attachment of bacterial cells was studied in QCM-D [41], is a more suitable model to describe biofilm growth in QCM-D.

In the context of the coupled resonance model, the QCM-D data can be interpreted as follows. Initially, as shown in Figure 3, both bacteria attached to the surface as single cells. The positive frequency shifts observed during this initial seeding step indicates that, initially, both strains were weakly coupled to the surface providing them with a resonance frequency below the 
sensor's third harmonic of $15 \mathrm{MHz}$. The pili-deficient $\Delta$ pilA mutant gave rise to a small positive $\Delta f$ and a small $\Delta D$ that remained constant through seeding, suggesting that its coupling to the surface remained weak throughout the first hour. The wild-type $P$. aeruginosa PAO1 strain gave rise to a large positive $\Delta f$ and a large $\Delta D$ that changed direction after $\sim 20$ min (while still being positive relative to the baseline). While $\Delta D$ eventually reached a plateau at a positive value of $\left(\sim 3 \times 10^{-6}\right)$, the frequency shift reached a small negative $\Delta f$ value $(\sim 3 \mathrm{~Hz})$. This transition from a positive to a negative frequency shift, accompanied with a peak in the dissipation shift, for $P$. aeruginosa PAO1, suggests that pili gradually transitioned the coupling of those bacteria to the surface such that their inherent resonance frequency increased to a value above the $15 \mathrm{MHz}$ of the third QCM-D harmonic (i.e., an increase in coupling constant $k$, according to equation 1).

This "bond maturation" process, which spanned over $\sim 30 \mathrm{~min}$, was important for the remainder of the experiments as it essentially determined how the biomass of subsequent biofilm growth coupled to, and thus was sensed by the QCM-D sensor. While for the wild-type $P$. aeruginosa $\mathrm{PAO} 1$ strain, both $\Delta f$ and $\Delta D$ increased in a non-linear fashion with increased biomass (i.e., an increase in mass $m$, according to equation 1), in comparison, both $\Delta f$ and $\Delta D$ barely changed with increased $P$. aeruginosa $\triangle$ pilA biomass (Figure 6). Specifically, the QCM-D sensor response to biomass accumulation during biofilm growth depends on how the biomass (i.e., the biofilm) is coupled to the surface. In this study, the difference in how the QCM-D responds to biomass accumulation of the two strains can be attributed to the presence or absence of pili cell surface appendages.

It should be noted that the frequency shift of the $\Delta$ pilA mutant was not always positive after the $20 \mathrm{~h}$ biofilm growth, but sometimes slightly negative (see variation in Figure 2). Since the $\Delta$ pilA did not show any signs of a dissipation peak during experiments, this is less likely a 
result of change in the bacteria's coupling with the sensor, but more likely caused by surface adsorption of bacteria-secreted extracellular polymeric substances which is concomitant with biofilm formation and has been shown to be able to shift the "baseline" towards negative values [36].

An interesting observation from this study is the recurrent fluctuation in both $\Delta f$ and $\Delta D$ signals (Figure 1), which appears to coincide with the microcolony dispersion and bacterium surface migration event that gradually saturated the surface with cells and occurred between 4 and $6 \mathrm{~h}$ after seeding (Figure 3). This is consistent with a previous study where P. aeruginosa was found to almost completely cover the substratum surface after $\sim 5$ to $7 \mathrm{~h}$ following inoculation [18] (identical to our 4 to $6 \mathrm{~h}$, following $1 \mathrm{~h}$ seeding; i.e., 5 to $7 \mathrm{~h}$ following inoculation). This fluctuation in the signal may be interpreted as a dynamic modification of the coupling of the wildtype $P$. aeruginosa $\mathrm{PAO} 1$ to the QCM-D surface during migration (i.e., attachment-detachment events or twitching motility). Twitching motility in $P$. aeruginosa results from the repeated extension, tethering, and retraction of type IVa pili [46]. It is possible that this fluctuation in the QCM-D signal represents a coordinated rearrangement of cells on the surface, dependent on the presence of pili, which allows the wild-type cells to form a confluent, sheet-like biofilm. The distinct difference in QCM-D response to the initial bacterial seeding step between the two strains illustrates the importance of individual bacterium surface-coupling behavior for the outcome of this study. It is also possible that, at later stages of biofilm development, the biomass of the uniformly grown sheet-like wild-type biofilm became even better coupled to the surface because pili-mediated surface migration distributed wild-type cells over the entire surface, allowing for a larger number of biofilm surface-attachment points as compared to the non-migrating pili-deficient strain. 
TIRF experiments (Figure 5) provided further evidence of a difference in the manner the two strains adhere to the surface. The wild-type P. aeruginosa PAO1 strain showed a greater increase in fluorescence intensity during its surface approach than the pili-deficient $P$. aeruginosa $\Delta$ pilA mutant strain, suggesting that pili-mediated adhesion brings the bacteria to closer contact with the surface than non-pili mediated adhesion. The bacteria's surface approach time constants were different; $0.5 \mathrm{~s}$ for the pili-deficient $P$. aeruginosa $\Delta$ pilA, compared to $1-2 \mathrm{~s}$ for the wild-type P. aeruginosa PAO1. Considering the $200 \mathrm{~nm}$ penetration depth of the evanescent light wave in the TIRF setup, the time constants determined for the pili-bearing $P$. aeruginosa PAO1 surface approach are similar to pili extension and retraction rates reported previously (from 500 to 2000 $\left.\mathrm{nm} \mathrm{s}^{-1}\right)[47,48,49]$. Although the TIRF data demonstrate a difference in the initial surface approach of these two strains, the time scales involved (i.e., few seconds) are order of magnitudes shorter than the "bond maturation" of 25 minutes observed in QCM-D for P. aeruginosa PAO1. Thus, the "bond maturation" observed in QCM-D cannot solely be explained by the bacteria's surface approach, but rather suggests that another more long-term, pili-governed mechanism must be at play. 


\section{Conclusions}

We have shown that biofilm growth can be monitored in real time using a QCM-D. While this has been demonstrated in earlier studies, we herein also demonstrate that the way the QCM-D responds to biofilm growth differs across different biofilm types. More specifically, the QCM-D response per unit biomass depends on how the biofilm couples to the surface, highlighting the applicability of QCM-D to sense the nature of biofilm attachment rather than biofilm growth. Pili-mediated biofilm attachment allowed for larger frequency and dissipation shifts per unit of biomass than non-pili-mediated biofilm attachment which, with support from TIRF bacterium fluorescence intensity data, is indicative of a stronger coupling with the surface, thus demonstrating the distinct role of pili in biofilm attachment mechanisms. 


\section{Acknowledgments}

A.L.J.O. and M.R.M. contributed to this work in equal parts. The authors acknowledge the financial support of the Natural Sciences and Engineering Research Council of Canada (Discovery Grant to N.T. and NSERC Strategic Research Network on Bioactive Paper SENTINEL), and the CRC Program. MRM acknowledges the Concordia Institute for Water, Energy and Sustainable Systems for a CREATE award and McGill University for the F. Grey Woods Fellowship. The authors also acknowledge A. Nieradka for graphics assistance. TIRF was conducted in the McGill University Life Sciences Complex Advanced BioImaging Facility (ABIF). 


\section{References}

[1] L. Chen and Y.M. Wen, International Journal of Oral Science, 3 (2011) 66.

[2] B. Carpentier and O. Cerf, Journal of Applied Bacteriology, 75 (1993) 499-511.

[3] A. Bridier, P. Sanchez-Vizuete, M. Guilbaud, J.C. Piard, M. Naïtali and R. Briandet, Food Microbiology, 45 (2015) 167-178.

[4] T.R. Lenhart, K.E. Duncan, I.B. Beech, J.A. Sunner, W. Smith, V. Bonifay, B. Biri and J.M. Suflita, Biofouling, 30 (2014) 823-835.

[5] L. Hall-Stoodley, J.W. Costerton and P. Stoodley, Nature Reviews Microbiology, 2 (2004) 95108.

[6] M. Rattier, J. Reungoat, J. Keller and W. Gernjak, Water Research, 54 (2014) 89-99.

[7] Y. Bordon, Nature Reviews Microbiology, 13 (2015) 4.

[8] J.B. Xavier, D.C. White and J.S. Almeida, Water Science \& Technology, 47 (2003) 31-37.

[9] E. Peeters, H.J. Nelis and T. Coenye, Journal of Microbiological Methods, 72 (2008) 157-165.

[10] A. Houari, J. Picard, H. Habarou, L. Galas, H. Vaudry, V. Heim and P. Di Martino, Biofouling, 24 (2008) 235-240.

[11] M. Whiteley, M.G. Bangera, R.E. Bumgarner, M.R. Parsek, G.M. Teitzel, S. Lory and E.P. Greenberg, Nature, 413 (2001) 860-864.

[12] K. Sauer, A.K. Camper, G.D. Ehrlich, J.W. Costerton and D.G. Davies, Journal of Bacteriology, 184 (2002) 1140-1154.

[13] H.H. Fang, K.Y. Chan and L.C. Xu, Journal of Microbiological Methods, 40 (2000) 89-97.

[14] C.J. Wright, M.K. Shah, L.C. Powell and I. Armstrong, Scanning, 32 (2010) 134-149.

[15] K.H. Simpson, M.G. Bowden, M. Höök and B. Anvari, Lasers in Surgery and Medicine, 31 (2002) 45-52.

[16] H.J. Busscher and H.C. van der Mei, Clinical Microbiology Reviews, 19 (2006) 127-141.

[17] T.R. Garrett, M. Bhakoo and Z. Zhang, Progress in Natural Science, 18 (2008) 1049-1056.

[18] G.A. O'Toole and R. Kolter, Molecular Microbiology, 30 (1998) 295-304.

[19] T.R. De Kievit, R. Gillis, S. Marx, C. Brown and B.H. Iglewski, Applied and Environmental Microbiology, 67 (2001) 1865-1873.

[20] M. Klausen, A. Aaes-Jørgensen, S. Molin and T. Tolker-Nielsen, Molecular Microbiology, 50 (2003) 61-68.

[21] M. Klausen, A. Heydorn, P. Ragas, L. Lambertsen, A. Aaes-Jørgensen, S. Molin and T. TolkerNielsen, Molecular Microbiology, 48 (2003) 1511-1524.

[22] G.Z. Sauerbrey, Journal der Physik, 155 (1959) 206-212.

[23] M.C. Dixon, Journal of Biomolecular Techniques, 19 (2008) 151.

[24] K. Otto, Research in Microbiology, 159 (2008) 415-422.

[25] D.E. Nivens, J.Q. Chambers, T.R. Anderson and D.C. White, Analytical Chemistry, 65 (1993) 65-69.

[26] H. Helle, P. Vuoriranta, H. Välimäki, J. Lekkala and V. Aaltonen, Sensors and Actuators B: Chemical, 71 (2000) 47-54.

[27] J. Kreth, E. Hagerman, K. Tam, J. Merritt, D.T.W. Wong, B.M. Wu, N. V. Myung, W. Shi and F. Qi, Biofilms, 1 (2004) 277-284. 
[28] A.L. Schofield, T.R. Rudd, D.S. Martin, D.G. Fernig and C. Edwards, Biosensors and Bioelectronics, 23 (2007) 407-413.

[29] M.Y. Chen, M.J. Chen, P.F. Lee, L.H. Cheng, L.J. Huang, C.H. Lai and K.H. Huang, Biochemical Engineering Journal, 53 (2010) 121-130.

[30] J.M. Kleijn, Q. Lhuillier and A.W. Jeremiasse, 79 (2010) 272-275.

[31] P. Castro, P. Resa, C. Durán, J.R. Maestre, M. Mateo and L. Elvira, IOP Conference Series: Materials Science and Engineering, International Symposium on Ultrasound in the Control of Industrial Processes, December 2012, IOP Publishing, 2012, p. 012054.

[32] K. Chen, D. Le, H. Zhang, L. Nie and S. Yao, Analytica Chimica Acta, 329 (1996) 83-89.

[33] A. Bressel, J.W. Schultze, W. Khan, G.M. Wolfaardt, H.P. Rohns, R. Irmscher and M.J. Schöning, Electrochimica Acta, 48 (2003) 3363-3372.

[34] V. Reipa, J. Almeida and K.D. Cole, Journal of Microbiological Methods, 66 (2006) 449-459.

[35] M. Ruhnow, J. Kohser, T. Bley, E. Boschke, M. Bulst and S. Wegner, (2014, April). IEEE Ninth International Conference on Intelligent Sensors, Sensor Networks and Information Processing (ISSNIP), Singapore, 21-24 April 2014, IEEE, p. 1-4.

[36] A.L.J. Olsson, H.C. van der Mei, D. Johannsmann, H.J. Busscher and P.K. Sharma, Analytical Chemistry, 84 (2012) 4504-4512.

[37] A.L.J. Olsson, H.C. van der Mei, H.J. Busscher and P.K. Sharma, Journal of Colloid and Interface Science, 357 (2011) 135-138.

[38] A. Pomorska, D. Shchukin, R. Hammond, M.A. Cooper, G. Grundmeier and D. Johannsmann, Analytical Chemistry, 82 (2010) 2237-2242.

[39] G.L. Dybwad, Journal of Applied Physics, 58 (1985) 2789-2790.

[40] A.L.J. Olsson, N. Arun, J.S. Kanger, H.J. Busscher, I.E. Ivanov, T.A. Camesano, Y. Chen, D. Johannsmann, H.C. van der Mei and P.K. Sharma, Soft Matter, 8 (2012) 9870-9876.

[41] I.M. Marcus, M. Herzberg, S.L. Walker and V. Freger, Langmuir, 28 (2012) 6396-6402.

[42] J.Y. Walz, Current Opinion in Colloid \& Interface Science, 2 (1997) 600-606.

[43] H.J. Busscher, W. Norde, P.K. Sharma and H.C. Van der Mei, Current Opinion in Colloid \& Interface Science, 15 (2010) 510-517.

[44] A. Granéli, J. Rydström, B. Kasemo and F. Höök, Biosensors and Bioelectronics, 20 (2004) 498504.

[45] G. Dunér, E. Thormann and A. Deddinaite, Journal of Colloid and Interface Science, 408 (2013) 229-234.

[46] L.L. Burrows, L. L. Annual Review of Microbiology, 66 (2012) 493-520.

[47] A.J. Merz, M. So and M.P. Sheetz. Nature, 407 (2000) 98-102.

[48] J.M. Skerker and H.C. Berg, Proceedings of the National Academy of Sciences, 98 (2001)

6901-6904.

[49] M. Clausen, M. Koomey and B. Maier. Biophysical Journal, 96 (2009) 1169-1177. 


\section{Figures}
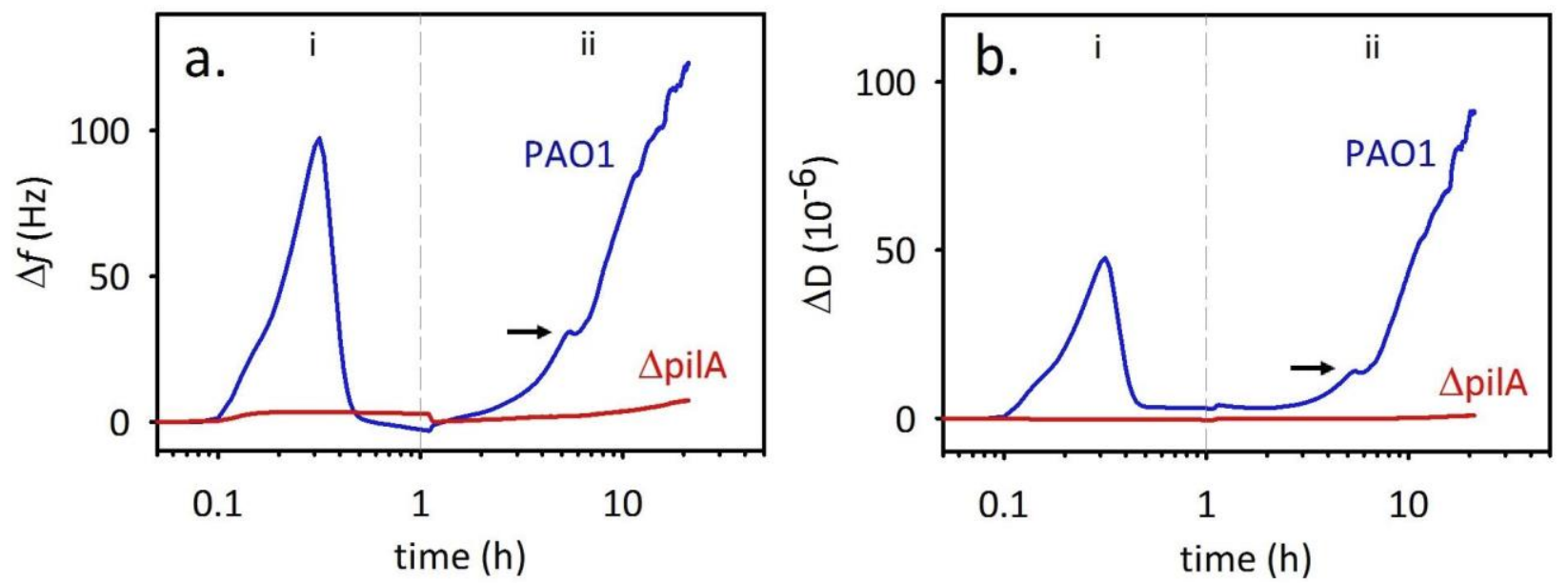

Figure 1. Representative (a) frequency shifts $(\Delta f)$ and (b) dissipation shifts $(\Delta D)$ of the $3^{\text {rd }}$ overtone during an initial seeding period (I) and the subsequent biofilm growth period (II) for wild-type $P$. aeruginosa PAO1 and the $\triangle$ pilA mutant. The arrows indicate the characteristic recurrent fluctuation in QCM-D signal observed between 4-6 h P. aeruginosa PAO1 biofilm growth. 

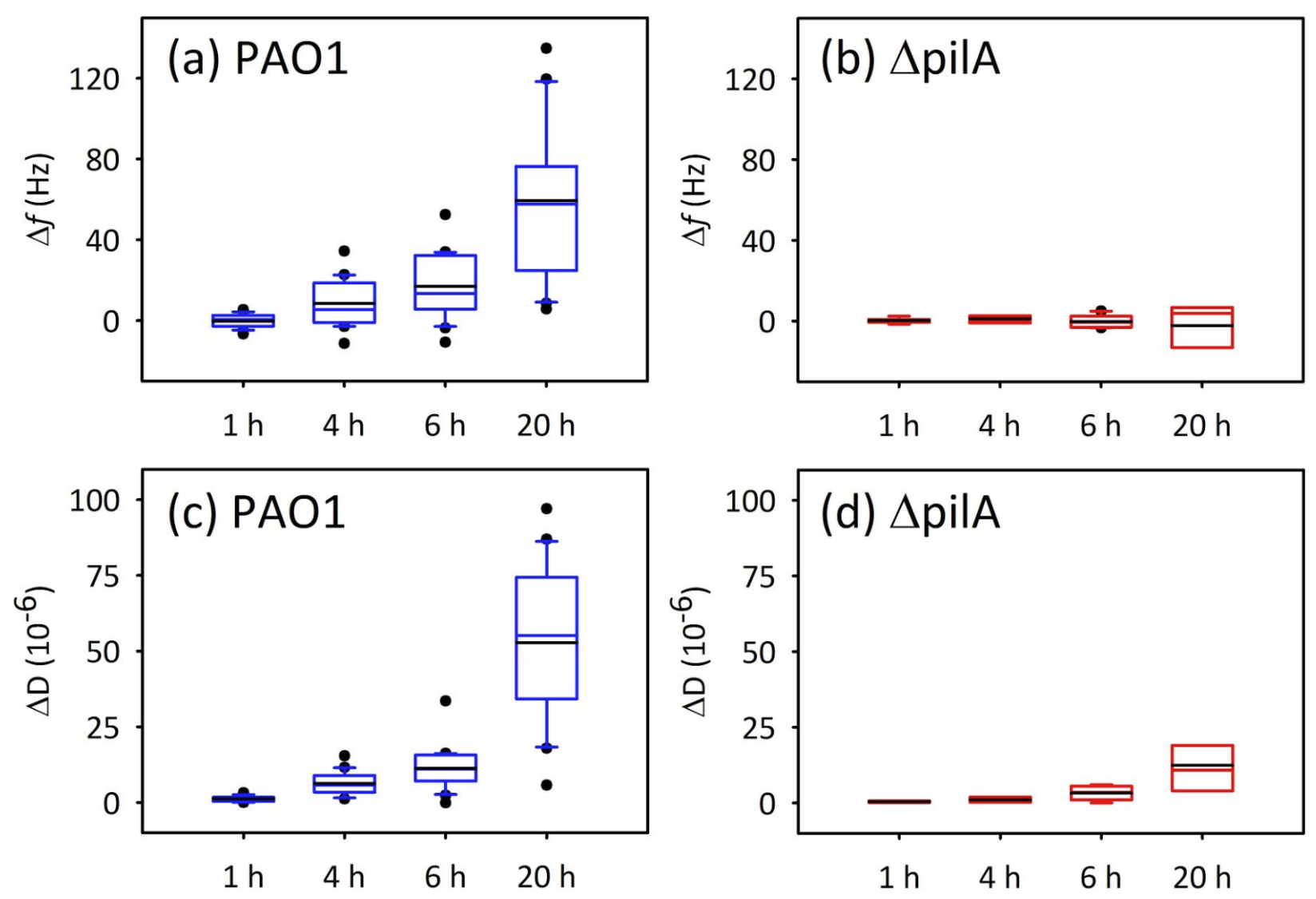

Figure 2. Box plots of values of $\Delta$ fand $\Delta D$ at four time points $(1 \mathrm{~h}, 4 \mathrm{~h}, 6 \mathrm{~h}$ and $20 \mathrm{~h}$ ) selected to coincide with CLSM imaging of biofilm growth. Black solid lines indicate average values and blue and red solid lines indicate median values for wild-type P. aeruginosa PAO1 and the $\triangle$ pilA mutant, respectively. 


\section{P. aeruginosa $\mathrm{PAO} 1$ wild-type}

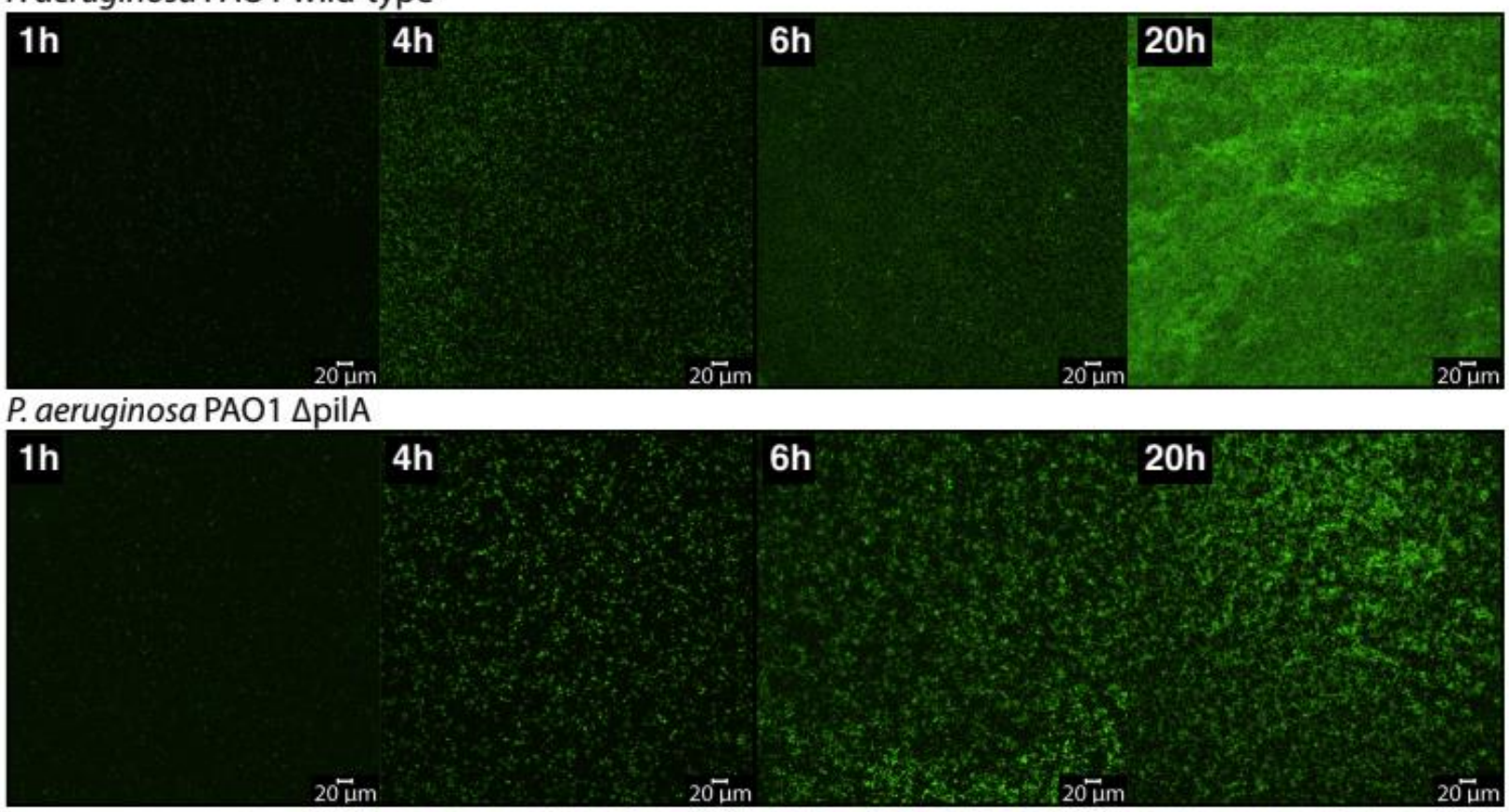

Figure 3. Representative maximum intensity projections of CLSM image stacks of (a) P. aeruginosa PAO1 and (b) $\triangle$ pilA mutant biofilm development on QCM-D crystals at four selected time points $(1 \mathrm{~h}, 4$ $h, 6 \mathrm{~h}$ and $20 \mathrm{~h})$.
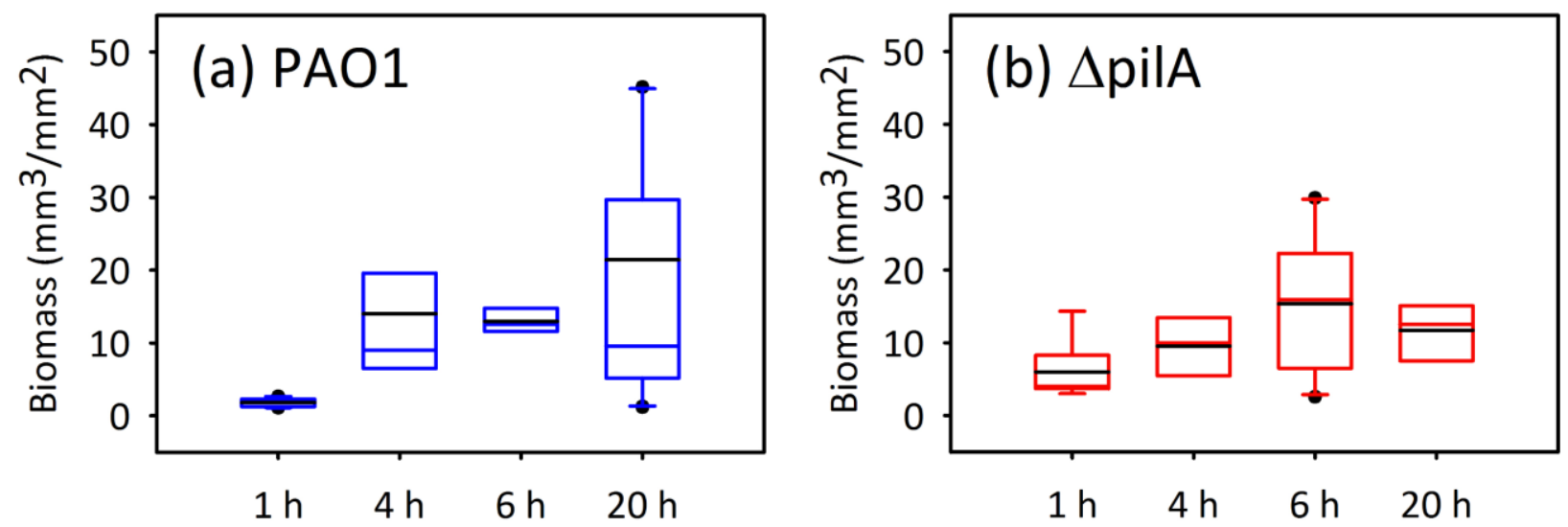

Figure 4. Box plots of biomass estimates determined using COMSTAT2 analysis of the CLSM images of (a) wild-type P. aeruginosa PAO1 and (b) $\triangle$ pilA mutant biofilm. Black solid lines indicate average values and blue and red solid lines indicate median values for wild-type P. aeruginosa PAO1 and the $\triangle$ pilA mutant, respectively. 

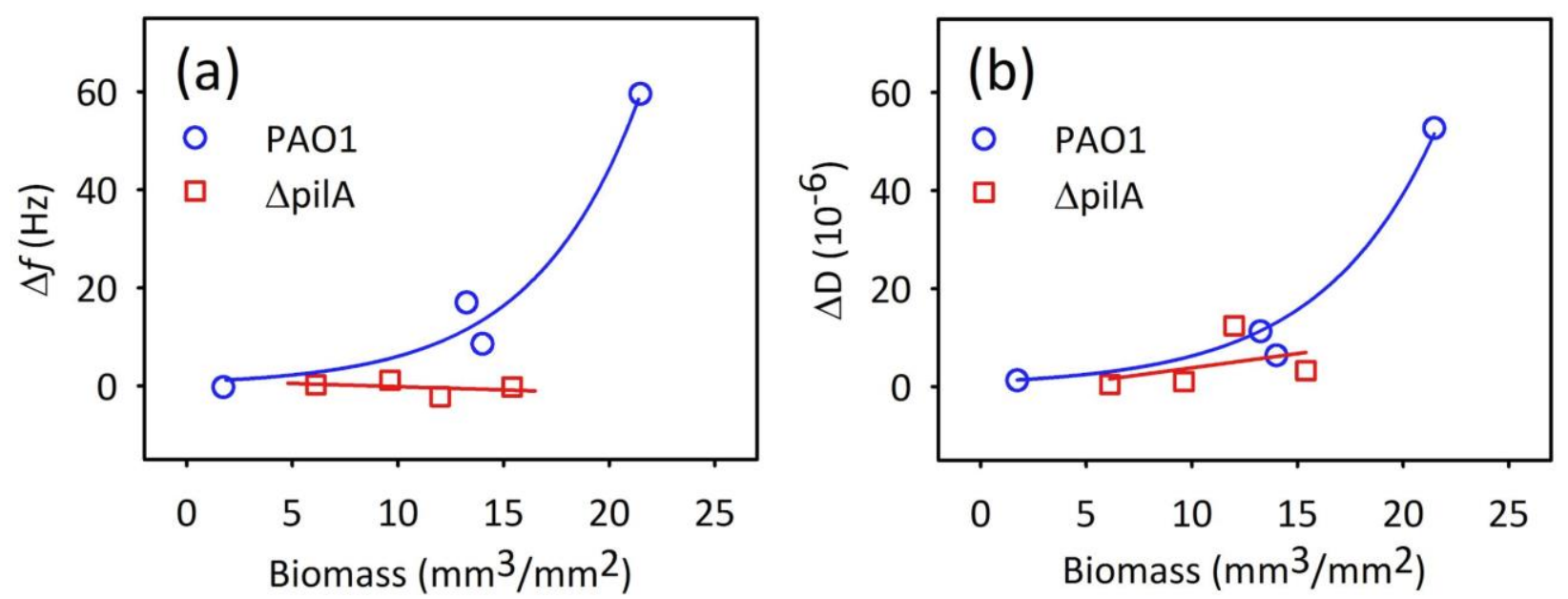

Figure 5. Average values of (a) $\Delta f$ and (b) $\Delta D$ plotted as a function of average biomass estimates at four time points $(1 \mathrm{~h}, 4 \mathrm{~h}, 6 \mathrm{~h}$ and $20 \mathrm{~h})$ for the wild-type P. aeruginosa PAO1 and $\triangle$ pilA mutant biofilms.

Data are obtained from average values in the boxplots presented in Figures 2 and 4. 

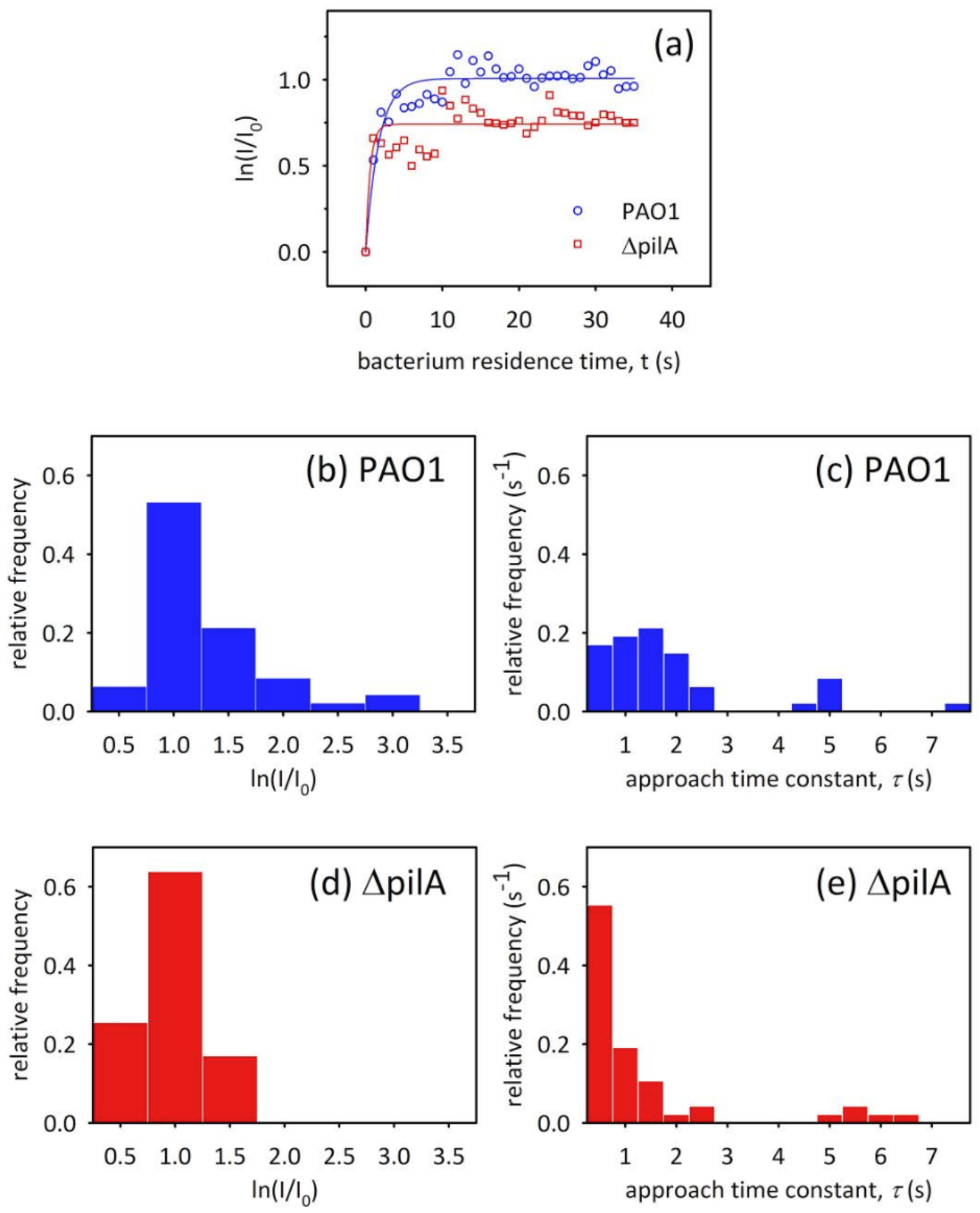

Figure 6. Summary of TIRF results depicting (a) representative examples of TIRF fluorescence intensities for individual wild-type P. aeruginosa PAO1 and $\triangle$ pilA mutant bacteria as a function of bacteriumsurface residence time, along with histograms of the final change in florescence intensity at infinity $\left(\ln \left(I / I_{0}\right)_{\infty}\right)$ and the approach time constants for individual ( $b$ and $c$ ) wild-type and (d and e) $\Delta$ pilA bacteria, respectively. 